\title{
Energy states, oscillator strengths and polarizabilities of many electron atoms confined by an impenetrable spherical cavity
}

\author{
yusuf yakar ${ }^{1}$, Bekir Çakır², and Ayhan Özmen³ \\ ${ }^{1}$ arts and sciences faculty \\ ${ }^{2}$ Selcuk University \\ ${ }^{3}$ Selcuk Universitesi Fen Fakultesi
}

September 11, 2020

\begin{abstract}
Abstract: The orbital, ground and excited state energies of many electron atoms confined by an impenetrable spherical cavity with radius R are calculated using Quantum Genetic Algorithm (QGA) approach and Hartree-Fock Roothaan (HFR) theory. The important properties such as static and dynamic polarizability, oscillator strength and static pressure are investigated as perturbative. The results reveal that cavity radius and impurity charge have played an important role on the polarizability, the oscillator strength and pressure of the system. In addition, it is seen that when cavity radius is extremely large, all energies and the other physical parameters approach the energies and physical parameters of unconfined atom. As the dot radius decreases, the polarizability of system because of the strong spatial confinement decreases, but the pressure exerting on the system as the cavity radius $\mathrm{R}$ is shrunk increases. In addition, as the impurity charge increases, the magnitude of the oscillator strength decreases. Keywords: Orbital energy, static and dynamic polarizability, oscillator strength, pressure, many electron quantum dots.
\end{abstract}

Energy states, oscillator strengths and polarizabilities of many electron atoms confined by an impenetrable spherical cavity

Yusuf Yakar ${ }^{1} 11^{*}$ Corresponding authorsTel: +903822882167 - Faks: +90 3822882299 E-mail address:yuyakar@yahoo.com (Y.Yakar)bcakir@selcuk.edu.tr(B.Çakır) Bekir Çakır ${ }^{2 *}$ Ayhan Özmen ${ }^{2}$

${ }^{1}$ Physics Department, Faculty of Arts and Science, Aksaray University, Campus 68100, Aksaray-Turkiye

${ }^{2}$ Physics Department, Faculty of Science, Selcuk University, Campus 42031, Konya-Turkiye

Abstract: The orbital, ground and excited state energies of many electron atoms confined by an impenetrable spherical cavity with radius $\mathrm{R}$ are calculated using Quantum Genetic Algorithm (QGA) approach and Hartree-Fock Roothaan (HFR) theory. The important properties such as static and dynamic polarizability, oscillator strength and static pressure are investigated as perturbative. The results reveal that cavity radius and impurity charge have played an important role on the polarizability, the oscillator strength and pressure of the system. In addition, it is seen that when cavity radius is extremely large, all energies and the other physical parameters approach the energies and physical parameters of unconfined atom. As the dot radius decreases, the polarizability of system because of the strong spatial confinement decreases, but the pressure exerting on the system as the cavity radius $R$ is shrunk increases. In addition, as the impurity charge increases, the magnitude of the oscillator strength decreases. 
Keywords: Orbital energy, static and dynamic polarizability, oscillator strength, pressure, many electron quantum dots.

\section{INTRODUCTION}

In the past decade, confined quantum systems are of great interest in many branches of physics, chemistry and engineering due to having unique properties and potential applications in microelectronic and optoelectronic devices. When atom is enclosed in a spherical cavity with impenetrable walls, atom's electrons are affected by a confining potential in at least one direction. Such small structures are often referred to as zero-dimensional structures or quantum dots, in which the charge carriers are confined in all three dimensions. Quantum dots (QDs) have discrete energy levels, like real atoms, and they are also often called artificial atoms. ${ }^{[1]}$ The energy levels and dot sizes can be controlled by adjusting the potential barrier. Thus, the quantum confinement effects cause drastic changes in observable properties of QDs. Technologically, QDs have many potential uses in microelectronic and optoelectronic devices. In this respect, by employing different approaches and potential shapes, some researchers have studied the electronic structure, ${ }^{[2-5]}$ binding energies, ${ }^{[6-8]}$ optical properties, ${ }^{[9-15]}$ electric and magnetic field effects, ${ }^{[16-25]}$ and other physical properties ${ }^{[26-30]}$ of single electron QDs. As well known, it is easy to obtain analytical solutions for QDs with one electron. However, analytical solutions of many electron QDs are more difficult, dull and complex than the single electron QDs. Researchers are used various approach methods to overcome these problems. Recently, several studies investigating the various properties of two electron QDs have been published. By employing the Kohn-Sham model, Aquino et al. ${ }^{[31]}$ calculated energy states of helium atom inside impenetrable spherical box. Wilson et al. ${ }^{[32]}$ performed the ground state energies for two electron atoms confined by an impenetrable spherical cavity. Ludeña ${ }^{[33]}$ and Garza et al. ${ }^{[34]}$ reported the Hartree-Fock energy results for the confined many electron atoms by utilizing Roothaan's approach. By using QGA and HFR method, the various excited energy states and ionization energies of two electron QDs with and without parabolic potential are calculated by our group. ${ }^{[35]}$ Similarly, for the confined helium atom, the three lowest S symmetry state energies were computed by Flores-Riveros et al. ${ }^{[36]}$ by employing perturbative and variational method. Sarsa and $\operatorname{Sech}^{[37]}$ and Sañu-Ginarte et al. ${ }^{[38]}$ studied the ground and excited energy states of the confined systems such as $\mathrm{He}, \mathrm{Li}$ and Catoms by using the variational Monte Carlo and direct variational method. In 2020, Martinez-Flores and Cabrera-Trujillo ${ }^{\text {[39] }}$ calculated the ground and excited state energies of confined Li -like atoms in an impenetrable spherical cavity. They implemented Slater's $X-\alpha$ approach in Hartree-Fock theory to obtain the excitation energy spectrum, and they assumed that the inner electrons do not see the outer electrons. To the best of our knowledge, there are few theoretical studies related to the energy spectrum, orbital energy, oscillator strength, static dipole polarizability and pressure induced by the cavity for $\mathrm{He}^{-}, \mathrm{Li}, \mathrm{Be}^{+}, \mathrm{B}^{++}$and $\mathrm{Be}$ atoms confined by an impenetrable spherical cavity. We have used QGA procedure and HFR method to calculate the energies and wave functions, and the other physical parameters are performed from perturbative calculations.

\section{THEORY}

We have considered lithium/lithium-like and beryllium dots. Dots are supposed to be spherical with an infinite potential barrier that confines all particles inside. The time-independent Schrödinger equation of such a system is given by

$\hat{H} \Psi=E \Psi,(1)$

where $\hat{H}$ is the electronic Hamiltonian operator corresponding to the sum of the kinetic energies plus the potential energies for all the particles in the system, E and $\Psi$ are eigenvalue and eigenfunction of the Hamiltonian operator. The Hamiltonian operator is given by

$$
\hat{H}=\sum_{i=1}^{N}\left[\frac{-\hbar^{2} \nabla_{i}^{2}}{2 m}-\frac{Z e^{2}}{4 \pi \varepsilon_{0} r_{i}}+\sum_{j=1}^{i-1} \frac{e^{2}}{4 \pi \varepsilon_{0} r_{\mathrm{ij}}}+V_{c}\left(r_{i}\right)\right]
$$

where $m$ and $\varepsilon_{0}$ are the mass of electron and dielectric constant of the cavity, $\mathrm{Z}$ is the impurity charge, 
$r_{i}$ are electron's position vectors and $r_{\mathrm{ij}}$ are the mutual distance between the $i$ th and $j$ th electrons, $N$ is electron number $(N=3,4)$. Here, the first sum on the right side describes the kinetic energy operators for the electrons, the second sum represents the Coulomb potential energy for the attraction between the electrons and impurity charge, the third one denotes the electron-electron repulsion plus exchange repulsion energy operators. The last term $V_{C}$ represents the confining potential term and its form is defined by

$$
V_{C}\left(r_{1}, r_{2}, r_{3}, r_{4}\right)=\{
$$

$$
\begin{gathered}
0, \quad \text { if } r_{1}, r_{2}, r_{3}, r_{4}<R \\
\infty, \quad \text { if } r_{1}, r_{2}, r_{3}, r_{4} \geq R,
\end{gathered}
$$

where $\mathrm{R}$ is confinement radius (or dot radius). In three and four-electron systems, for the ground state configurations $1 s^{2} 2 s$ and $1 s^{2} 2 s^{2}$, the total wavefunction of the system is expressed by the Slater determinant including both space and spin variables

$$
\Psi=\left\{\begin{array}{lr}
\left|\phi_{1 s}(1) \phi_{1 s}(2) \phi_{2 s}(3)\right| & \text { for } N=3 \\
\left|\phi_{1 s}(1) \phi_{1 s}(2) \phi_{2 s}(3) \phi_{2 s}(4)\right| & \text { for } N=4
\end{array}\right.
$$

where $\phi$ represents the one-electron wave functions consisting of spin and spatial components. The spatial part of the wavefunction can be written as the linear combination of Slater type orbitals (STOs, $\chi$ ) as follows

$$
\phi_{i}=\sum_{k=1}^{\sigma} c_{\mathrm{ik}} \chi_{k}\left(\zeta_{k}, \mathbf{r}\right)
$$

where $\sigma$ is the number of basis sets, $c_{\mathrm{ik}}$ is the expansion coefficients and $\zeta_{k}$ is the orbital exponents and $k \rightarrow$ $n_{k} l_{k} m_{k}$ denotes the quantum numbers of basis sets. STOs are preferred in the quantum mechanical analysis of the electronic structure of confined and unconfined systems, because STOs represent more correct behavior of the electronic wavefunctions, especially important in the regions very close to or far from the impurity. Since the Hamiltonian in Eq.(2) does not include spin terms, the total energy is unaffected by inclusion of spin factor in the wave functions. In the HF approximation, the total energy is a sum of one-electron and two-electron energies. For a three and four electron system, the ground state energy is written

$$
\begin{array}{lr}
E=\{ & \\
2 \epsilon_{1 s}+\epsilon_{2 s}+J_{1 s 1 s}+(2 J-K)_{1 s 2 s} & \text { for } \quad N=3 \\
2 \epsilon_{1 s}+2 \epsilon_{2 s}+J_{1 s 1 s}+J_{2 s 2 s}+2(2 J-K)_{1 s 2 s} & \text { for } \quad N=4,
\end{array}
$$

where $\epsilon_{i}$ denotes the orbital energies and it is given by

$$
\begin{array}{ll}
\epsilon_{1 s}=\{ & \\
h_{1 s}+J_{1 s 1 s}+\left(J-\frac{1}{2} K\right)_{1 s 2 s} & \text { for } N=3 \\
h_{1 s}+J_{1 s 1 s}+(2 J-K)_{1 s 2 s} & \text { for } N=4,
\end{array}
$$

and

$$
\begin{array}{lr}
\epsilon_{2 s}=\{ & \\
h_{2 s}+(2 J-K)_{2 s 1 s} & \text { for } N=3 \\
h_{2 s}+J_{2 s 2 s}+(2 J-K)_{2 s 1 s} & \text { for } N=4,
\end{array}
$$

in which $h$ is one-electron energy integrals (kinetic energy plus impurity attraction energy), $J_{\mathrm{ij}}$ and $K_{\mathrm{ij}}$ show two-electron Coulomb and exchange energy integrals. Here, in the excited states, the valence electron in the 
$2 \mathrm{~s}$ sublevel is promoted to the nl $\alpha$ sublevel, that is $2 s=n l \alpha$. The integrals in Eqs. $(6,7)$ can be expressed over STOs as follows, in atomic units (au),:

$$
\begin{aligned}
h_{p}= & \left\langle\phi_{p}(\mathbf{r})\left|-\frac{\nabla^{2}}{2}-\frac{Z}{r}\right| \phi_{p}(\mathbf{r})\right\rangle=\sum_{i=1}^{\sigma} \sum_{j=1}^{\sigma} c_{\mathrm{pi}} c_{\mathrm{pj}}\left\langle\chi_{p_{i}}\left|-\frac{\nabla^{2}}{2}-\frac{Z}{r}\right| \chi_{p_{j}}\right\rangle, \\
J_{p, q}= & \left\langle\phi_{p}\left(\mathbf{r}_{1}\right) \phi_{q}\left(\mathbf{r}_{2}\right)\left|\frac{1}{r_{12}}\right| \phi_{p}\left(\mathbf{r}_{1}\right) \phi_{q}\left(\mathbf{r}_{2}\right)\right\rangle \\
& =\sum_{i=1}^{\sigma} \sum_{j=1}^{\sigma} \sum_{s=1}^{\sigma} \sum_{k=1}^{\sigma} c_{\mathrm{pi}} c_{\mathrm{qi}} c_{\mathrm{ps}} c_{\mathrm{qk}}\left\langle\chi_{p_{i}} \chi_{q_{j}}\left|\frac{1}{r_{12}}\right| \chi_{p_{s}} \chi_{q_{k}}\right\rangle,(8 \mathrm{~b})
\end{aligned}
$$

and

$$
\begin{aligned}
K_{p, q}= & \left\langle\phi_{p}\left(\mathbf{r}_{1}\right) \phi_{q}\left(\mathbf{r}_{2}\right)\left|\frac{1}{r_{12}}\right| \phi_{q}\left(\mathbf{r}_{1}\right) \phi_{p}\left(\mathbf{r}_{2}\right)\right\rangle \\
& =\sum_{i=1}^{\sigma} \sum_{j=1}^{\sigma} \sum_{s=1}^{\sigma} \sum_{k=1}^{\sigma} c_{\mathrm{pi}} c_{\mathrm{qi}} c_{\mathrm{ps}} c_{\mathrm{qk}}\left\langle\chi_{p_{i}} \chi_{q_{j}}\left|\frac{1}{r_{12}}\right| \chi_{q_{k}} \chi_{p_{s}}\right\rangle
\end{aligned}
$$

One- and two-electron integrals can be easily evaluated by modifying for appropriate consideration of the boundaries the expressions of atomic systems. ${ }^{[40]}$

In optical transitions between energy levels, the transition probability of absorption or emission of electromagnetic radiation is defined by oscillator strength. The oscillator strength (OS) is a dimensionless quantity and it plays an important role in spectroscopic studies. This term determines the intensity of a specific spectral line in atomic spectrum and also offers additional information on the fine structure. ${ }^{41}$ OS is given by, in au,

$f_{\gamma \rightarrow n}=2\left(E_{n}-E_{\gamma}\right)\left|\left\langle M_{\nu \gamma}\right\rangle\right|^{2}$,

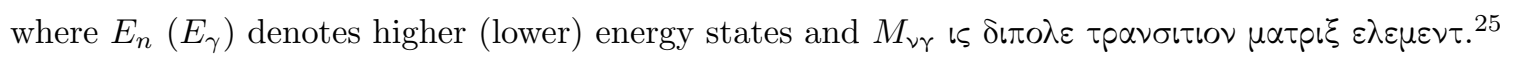

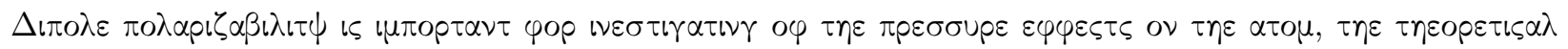

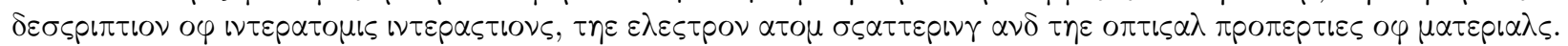

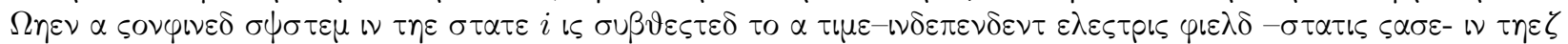

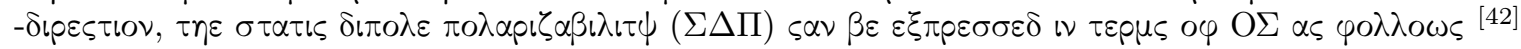

$\alpha_{\gamma}=\sum_{n \neq \gamma} \frac{f_{\gamma \rightarrow n}}{\left(E_{n}-E_{\gamma}\right)^{2}}$,

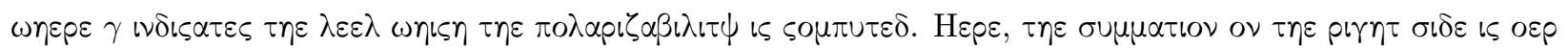

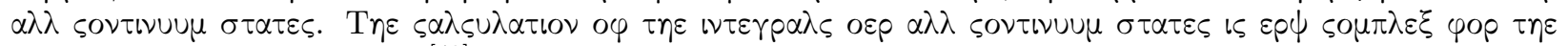

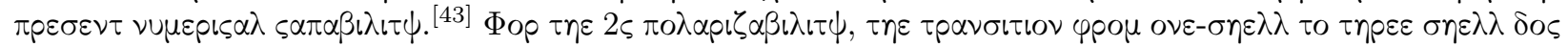

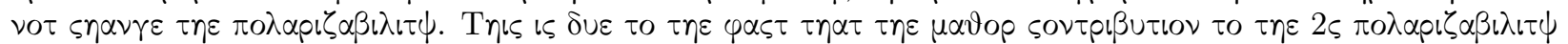

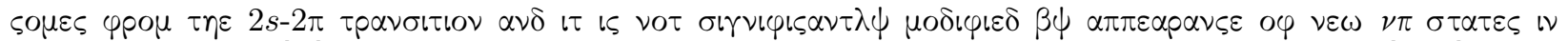

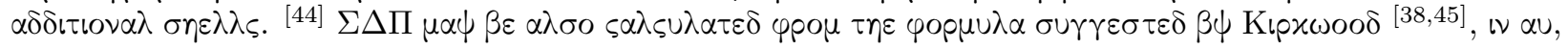

$\alpha_{i}^{K}=\frac{4}{9} \sum_{i=1}\left\langle r_{i}^{2}\right\rangle^{2}$

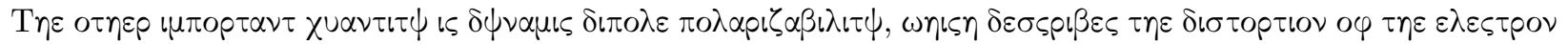

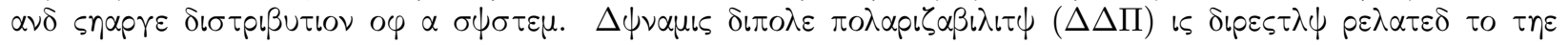

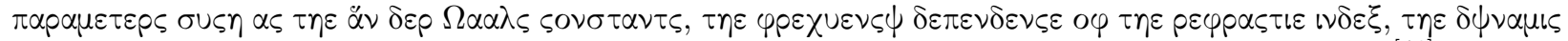

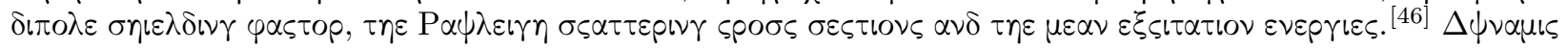

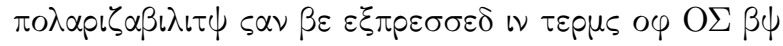

$\alpha(\nu)=\sum_{n>\gamma} \frac{f_{\gamma \rightarrow n}}{\left(E_{n}-E_{\gamma}\right)^{2}-n \nu^{2}}$.

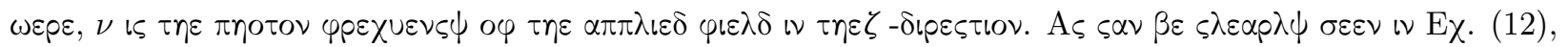

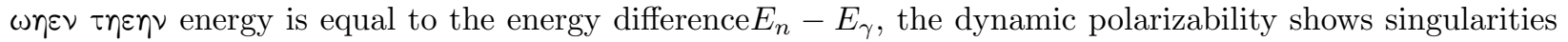
for any electronic state $n$. This is particularly important in cavities where the symmetry breaking due to confinement leads to very close-nearly degenerate- atomic levels. ${ }^{[44]}$ 
The compression of an atomic system leads to an increase in kinetic energy $K$ as a function of pressure $P$ . As the confinement radius $\mathrm{R}$ decreases, the average pressure that the cavity implements on the atomic system is given by the virial theorem as ${ }^{[33,47]}$

$K(R)=4 \pi R_{0}^{3} P(R)-E(R)$. (13)

\section{RESULTS AND DISCUSSION}

We have calculated the ground and excited state energies and wavefunctions of lithium/lithium-like and beryllium atoms confined at the centre of an impenetrable spherical cavity of dot radius $\mathrm{R}$. To minimize the total energy over STOs we have used the new variational method, which is a combination of QGA procedure and HFR method. In order to maintain the orthogonality of orbital, we have used the same set of screening parameters for all the one-electron spatial orbital with the same angular momentum and employed seven basis sets to calculate the energy expectation value. The calculations are made considering a single Slater determinant configuration. Therefore, the electron correlation effects being the second-order and higher order effects are not taken into account. Our results are given in terms of au (Hartree), and these results include terms with a fixed magnetic quantum numbersm (i.e., $m=m^{\prime}=0$ ).

In Table 1 and Table 2 show the orbital energies and total energies for the confined Lithium atom's electronic configurations $1 s^{2} 2 s\left({ }^{2} \mathrm{~S}\right)$ and $1 s^{2} 2 p\left({ }^{2} \mathrm{P}\right)$ for various dot radii. As seen in tables, both the orbital energy and the total energy increase as the confinement radius $\mathrm{R}$ decreases. In Table 1 , in all confinement regions, our results are in good agreement with the results calculated by Ludeña. ${ }^{[33]}$ However, there are small discrepancies between the literature results obtained by Flores and Trujillo. ${ }^{[39]}$ This difference is slightly increase in 1sorbital energy $\epsilon_{1 s}$. This is originated from their calculation method. They assume that the inner electrons do not see the outer electron in the electronic configuration $1 s^{2} 2 s$. It can be remarked that the confinement radius becomes very large, all energy states approach the corresponding states of unconfined lithium atom. For example, for the ground state, at $\mathrm{R}=10$, we have obtained the results as $\epsilon_{1 s}=-2.47672 a u, \epsilon_{2 s}=$ $-0.19366 a u$ and $E_{1 s^{2} 2 s}=-7.42992 a u$. Unconfined Lithium atom's orbital and ground state energies are $\epsilon_{1 s}=$ $-2.477739 a u, \epsilon_{2 s}=-0.196321 a u$ and $E_{1 s^{2} 2 s}=-7.432723 a u$. ${ }^{40}$ Similarly, for the first excited state, in Table 2 , at $\mathrm{R}=10$, we have obtained the results as $\epsilon_{1 \mathrm{~s}}=-2.50153 \mathrm{au}, \epsilon_{2 p}=-0.12788 \mathrm{au}$ and $E_{1 s^{2} 2 p}=$ $-7.37611 a u$. The literature value of $E_{1 s^{2} 2 p}$ is $-7.3865 a u .{ }^{[48]}$ It is noted that all energies increase when the spatial confinement is stronger. This is the result of the Heisenberg uncertainty principle. Similarly, for the excited state $1 s^{2} 2 p$, total energy results are better than the results obtained by Sañu-Ginarte. However, there are slight differences between our results and the results of Martinez et al. due to the reason explained above.

In Tables 3-5, we have illustrated the orbital energies, the ground and excited states energies for the confined $\mathrm{He}^{-}, \mathrm{Be}^{+}, B^{++}$and $\mathrm{Be}$ atoms. As seen from tables, when the dot radius becomes very large, the orbital and total energy levels approach the values of a free space atoms. For example, in Table 3 , at $\mathrm{R}=10$, for $1 s^{2} 2 s$ and $1 s^{2} 2 p$ configurations of $\mathrm{Be}^{+}$dot, we have obtained the numerical values as $\epsilon_{1 s}=-5.13779 \mathrm{au}, \epsilon_{2 s}=$ $-0.66581 \mathrm{au}, E_{1 s^{2} 2 s}=-14.27373 \mathrm{au}$ and $\epsilon_{1 s}=-5.15158 \mathrm{au}, \epsilon_{2 p}=-0.51796 a u, E_{1 s^{2} 2 p}=-14.12896$, respectively. The literature values for the excited state of unconfined $\mathrm{Be}^{+}$atom are $\epsilon_{1 s}=-5.1383425, \quad \epsilon_{2 s}=-0.6661462$ and $E_{1 s^{2} 2 p}=-14.277394 a u .{ }^{[49]}$ We have also showed the orbital and ground state energies of Be dot in Table 3 . The same situations are seen here. That is, as the dot radius increases, all energies approach the values that are equal to the corresponding energies of Be atom. For the orbital and ground state energy for Be dot, our results are consistent with the results of Ludeña and are lower than Sanu-Ginarte. In Table 4, we have illustrated the orbital, ground and excited state energies of $B^{++}$dot. It is worth to note that, while $R \gtrsim 1.5$, the speed of energy change is slowing down due to the strong attractive force. There are slight differences between our results and the literature results. On the other hand, in table 5 , for $\mathrm{He}^{-}$dot, the similar behaviours are obtained here. We could not compare our results with the literature data.

In Fig.1, we have plotted the ground $\left(1 s^{2} 2 s,{ }^{2} \mathrm{~S}\right)$ and excited $\left(1 s^{2} 2 p,{ }^{2} \mathrm{P}\right)$ state energies of the confinedHe ${ }^{-}, \quad L i, \mathrm{Be}^{+}, B^{++}$atoms as a function of dot radius. For each $n l$ state, as the confinement radius $R$ decreases, the energies increase more quickly. It is seen that when the confinement radius is extre- 
mely small (in the strong confinement region $R \lesssim 1.5$ ), the spatial confinement has a very strong influence on the impurity energy states. In this region, when the confinement radius continuously decreases, the wavefunctions of the localized states penetrates into the outside region of the quantum dot more and more. Therefore, the energies become leger and larger. On the other hand, in this region, the degeneracy of the energy spectrum is completely disappeared, and the energy states separate from each other. For the same principle quantum number, as the dot radius decreases, the energy of the level with smaller $l$ increases more quickly than the bigger $l$ level. The reason is that, most of time the confined electron in small $l$ level distributes itself at the more outer part of the impurity than the electron in big $l$ level.

In Fig.2, we display the orbital energies $\epsilon_{1 s}, \epsilon_{2 s}$ and $\epsilon_{2 p}$ of the confined lithium-like atoms as a function of the confinement radius $\mathrm{R}$. As it will be seen in (a), when the impurity charge $\mathrm{Z}$ increases, the orbital energy $\epsilon_{1 s}$ increases as negative. The energy $\epsilon_{1 s}$ becomes negative while $\mathrm{R} \gtrsim 2.3, \mathrm{R} \gtrsim 1.1, \mathrm{R} \gtrsim 0.65$ and $\mathrm{R} \gtrsim$ $0.5 \mathrm{forHe}^{-}, \quad L i, \mathrm{Be}^{+}$and $B^{++}$, respectively. Here, the negative sign indicates that we need to give the electron energy to move it from the confined atom. In the weak confinement region $R \geq 2.5$, while the spatial confinement effect on the energy $\epsilon_{1 s}$ is very weak, this effect becomes significant in the region $R \leq 1$, in which is known as the strong confinement region. As for in (b), similarly, the orbital energies $\epsilon_{2 s}$ and $\epsilon_{2 p}$ increase with the decrease of confinement radius until reaching the continuum. It is seen that while going to large dot radii, $\epsilon_{2 s}$ (dashed line) is deeper than the $\epsilon_{2 p}$ (solid line). As seen in (b), $\epsilon_{2 s}$ and $\epsilon_{2 p}$ energy states have the crossing points, which are highlighted by circles. These crossing points (or critical cavity radius) are at $R \sim 4.9, \quad R \sim 3.2, R \sim 2.4$ and $R \sim 2$ for $\mathrm{He}^{-}, \quad L i, \mathrm{Be}^{+}$and $B^{++}$dots, respectively. For the critical dot (cavity) radii, our results are in good agreement with the results reported by Flores and Trujillo [39], which are 3.4, 2.5 and 2.1 for $L i, \mathrm{Be}^{+}$and $B^{++}$, respectively. As the confinement radius is reduced, after these crossing points, this situation is exactly reversed, that is, $\epsilon_{2 p}$ energy state becomes higher than $\epsilon_{2 s}$ state. It is worth nothing that since the 2s-orbital is occupied with an electron in the initial ground state electronic configuration, for cavity with $\mathrm{R}$ lower than the critical cavity radius, one would have photon emission instead of absorption for initial electronic configuration. Thus, the excited electron from the initial $s$ - level to the final $p$ - level returns to ground state by the photon emission. That is, as the dot radius decreases, if the $p$ -level lies energetically below the corresponding $s$-level, this transition occurs. This situation is mostly seen in dipole oscillator transitions, and the oscillator strength become positive for excitations. As seen in $\epsilon_{2 s}$ and $\epsilon_{2 p}$ orbital energy curves, the crossing points shift toward smaller dot radii as the impurity charge $\mathrm{Z}$, and in large dot radii the difference between $\epsilon_{2 s}$ and $\epsilon_{2 p}$ orbital energies increases with the increase of Z .

Fig. 3 shows the dipole oscillator transition $1 \mathrm{~s}^{2} 2 \mathrm{~s} \rightarrow 1 \mathrm{~s}^{2} 2 \mathrm{p}$ for $L i, \quad \mathrm{Be}^{+}$and $B^{++}$dots as a function of R. For all dots, the oscillator curves exhibit similar behaviors. ForLi dot, in large dot radii, which is known as the weak confinement region, as the confinement radius decreases, the OS begins to reduce as positive until $\mathrm{R} \approx 3.2$, in which the crossing point occurs, and then it continues to decrease as negative until reaching to a negative constant. For $\mathrm{Li}$ dot , at $\mathrm{R}=10$, we find a value of $f_{2 S \rightarrow 2 P}=0.7489$. The literature value of unconfinedLi atom is $0.7488 .{ }^{[50]}$ As mentioned above, while $\mathrm{R} \gtrsim 3.2$, while the oscillator transition $f_{2 S \rightarrow 2 P}$ occurs by the photon absorption, it occurs by the photon emission after $\mathrm{R} \lesssim 3.2$. For $\mathrm{Be}^{+}$and $B^{++}$dots, we have observed similar behaviors. That is, the OS rapidly increases as the dot radius increases and then reaches to a limit value in larger dot radii. At $\mathrm{R}=10$, we have obtained the values of $f_{2 s \rightarrow 2 p}=0.5337$ and 0.4102 for the $\mathrm{Be}^{+}$and $B^{++}$ dots. The literature result of unconfinedBe ${ }^{+}$atom is $0.5505 .{ }^{[51]}$ Similarly, the oscillator transitions become negative after $\mathrm{R} \approx 2.4$ and $\mathrm{R} \approx 1.95$ for $\mathrm{Be}^{+}$and $B^{++}$dots, near the radii for which the crossing points occur. While $\mathrm{R}$ is smaller than the crossing points, in which point $\epsilon_{2 p}$ is lower than $\epsilon_{2 s}$, the oscillator transitions become by photon emission induced by the pressure cavity. As seen on the OS curves, the impurity has a strong effect on the oscillator transitions. The OS decreases with the increase of $\mathrm{Z}$ and shifts toward the smaller dot radius. The reason is that as the impurity charge increases, electrons are strongly attracted toward impurity. The similar results are obtained by Flores and Trujillo. ${ }^{[39]}$

In Fig.4, we have displayed the SDP of $L i, \mathrm{Be}^{+}$and $B^{++}$dots as a function of dot radius. For $2 \mathrm{~s}$ orbital, the SDP has been calculated from the Kirkwood formula in (a) and the oscillator strength in (b). As seen in (a), in large dot radii, it is worth nothing that the static polarizability of neutral Li dot is higher than the others since the electron cloud is deformed easily by the applied field. For Li dot, the static polarizability 
is very weak until $R \sim 3$, and more after it increases monotonically first up to $R \sim 12$ and then reaches a saturation value in large dot radii. In the strong confinement region, as the dot radius is reduced, the static polarizability decreases due to the fact that the localization causes a less polarizability of the charge distribution. When compared the SDP of $\mathrm{Li}$ with the SDP ofBe ${ }^{+}$and $B^{++}$, the effect of impurity charge $\mathrm{Z}$ is clearly seen on the SDP curves. As the $\mathrm{Z}$ increases, the polarizability rapidly decreases since the charge cloud cannot be easily deformed. In other words, the polarizability is associated with the binding energy of the electron. When the electrons get closer to impurity, the electron's binding energy increases, and thus the polarizability of the system starts to reduce due to increasing the binding energy. The opposite is also true. That is, when the binding energy of the electron is very weak, in very large dot radii, the polarizability becomes maximum. By using Kirkwood formula, for $\mathrm{R}=15$, we have obtained the polarizability values as 171.7730au, 16.9604au and 4.5015au for $\mathrm{Li}, \mathrm{Be}^{+}$and $\mathrm{B}^{++}$dots. The literature values for unconfined $\mathrm{Li}$ atom are 164au. ${ }^{[52]}$ In (b), in which the SDP has been calculated from the oscillator formula, for neutral Li dot, as the confinement radius reduces, the static polarizability decreases until reaching the crossing point $R \cong 3.2$. It is seen that static polarization changes its sign at the critical dot radius, $R \cong 3.2$. We have calculated the $\mathrm{SDP}$ values as $-63.54585 \mathrm{au}$ for $\mathrm{R}=3$ and 52.80064 au for $\mathrm{R}=3.5$. After the critical dot radius $R \cong 3.2$, as the confinement radius decreases, the polarizability approaches zero from negative value. Similar behaviors are seen on $\mathrm{Be}^{+}$dot in (b). The static polarizability of $\mathrm{Be}^{+}$dot is smaller than that of neutralLi. The sign of $\mathrm{SDP}$ in $\mathrm{Be}^{+}$dot changes at the critical dot radius $R \cong 2.3$. When compared $\mathrm{Li}$ withBe ${ }^{+}$, as the impurity charge increases, the critical dot radius at which SDP changes its sign shifts toward smaller dot radii. For $\mathrm{R}=15$, we have calculated the SDP values as 167.6403au and 27.2986au for $\mathrm{Li}$ and $\mathrm{Be}^{+}$dots. The literature values are 171.188au and 27. 3836au for unconfined Liand Be ${ }^{+}$atoms. ${ }^{[39]}$

Fig.5 shows that the dynamic dipole polarizability for Li andBe dots as a function of photon energy at $\mathrm{R}=1$, 1.5 and 2. The frequency step interval is taken as 0.01au. Singularities (or jumps) appearing on dynamic polarizability curves describe the frequency corresponding the energy difference in Eq.(12). As can be seen from Eq.(12), as the photon energy hv increases, the DDP increases until singularity point, in which point is ath $\nu=E_{n}-E_{\gamma}$. The presence of this pole leads to the sign inversion of the polarizability. It should be noted that the SDP peak's magnitude and its position which is equal to the singularity frequencies vary continuously according to the confinement radius. When compared $\mathrm{Li}$ with $\mathrm{Be}^{+}$, for a fixed $\mathrm{R}$, as the impurity charge increases, the peak positons of the DDP shift toward smaller photon energy.

The numerical values of kinetic energy and pressure that the cavity exerts on the system as the dot radius $\mathrm{R}$ (or cavity radius) is shrunk are presented in Table 6 and also the change of pressure and kinetic energy is displayed in Fig. 6 as a function of the cavity radius $\mathrm{R}$ for $L i, B^{++}$and Be dots. As seen in Table 6 and Fig.6, in weak confinement region or large dot radii, the pressure is very weak. As the dot radius decreases, both the pressure and the kinetic energy start to increase monotonically. In the strong confinement region, the smaller the dot radius, the higher the pressure will be. It can be said that the pressure is associated with the static polarizability. That is, in the strong confinement region, while the polarizability is very weak, the pressure is large. When the cavity radius is very large, in the weak confinement region, the pressure is very weak, but the polarizability becomes maximum. On the other hand, As the cavity radius decreases, both the pressure and the kinetic energy increases. According to the uncertainty principle, as the electron approaches the impurity, the uncertainty in the speed of the electron increases and so its kinetic energy increases. As a result of this, the pressure increases. It is also worthwhile to note that, for the same $\mathrm{R}$, the pressure is the highest for Be, decreases forLi and is the lowest for $B^{++}$. This is because the beryllium atom is more diffuse in its $2 \mathrm{~s}$ orbital according to the others, so the same cavity radius induces a high pressure. However, for the $B^{++}$, owing to increasing impurity charge, the boron ion has already compacted its 2 s electron, so the same cavity radius induces a smaller pressure on the ionic system. ${ }^{[39]}$ On the other hand, when compared Li with $B^{++}$, the effect of impurity charge on pressure is clearly seen here. That is, for the same confinement radius $\mathrm{R}$, the pressure of $B^{++}$is lower than the pressure of Li. However, for kinetic energy, the situation is opposite. For Be dot, our results are consistent with the literature results. ${ }^{[38]}$

\section{CONCLUSIONS}


In this work, we have calculated the ground and excited state energies and the orbital energies of three and four electron QDs such asHe ${ }^{-}, L i, \mathrm{Be}^{+}, B^{++}$and Bedots. We also carried out the static and dynamic dipole polarizability, oscillator strength and pressure induced by the cavity as a function of dot radius and impurity charge. The results show that both dot radius and impurity charge have a great influence on total energy, orbital energy, polarizability, oscillator strength and pressure of the system. While the polarizability is very weak due to the strong spatial confinement in small dot radii, it increases monotonically while going to large dot radii and then reaches a saturation value. For the ground state polarizability, the approximation of Kirkwood in many electron dots gives good results. It is found that while the oscillator transition $f_{2 s \rightarrow 2 p}$ occurs by the photon absorption until a critical dot radius, it occurs by the photon emission after the critical dot radius. As the dot radius decreases, both the pressure and the kinetic energy increases, but polarizability of the system decreases. To our knowledge, there are very little reports including the calculation of orbital energies, static polarizability, oscillator strength and pressure of many electron QDs. With respect to the lack of such studies, we believe that our study makes an important contribution to the literature. Also, theoretical investigation of electronic and optical properties of many electron QDs will lead to a better understanding of the properties of low dimensional structures. Such theoretical studies may have profound consequences about practical applications of the spectroscopic studies, and the results of this study will contribute to the research on related subjects.

\section{REFERENCES}

[1] Z.H. Zhang, G.C. Zhuang, K.X. Guo, J.H. Yuan, Superlatt. Microstruct. 2016, 100 , 440

[2] F.K. Boz, B. Nisancı, S. Aktas, S.E. Okan, Appl. Surface Science 2016, 387, 76;

S. Yılmaz, M. Kırak, Int. J. Mod. Phys. B 2018, 32, 1850154.

[3] R.L.M. Melono, C.F. Lukong, O. Motapan, J. Phys. B: At. Mol. Opt. Phys. 2018,51, 205005.

[4] G. Safarpour, M.A. Izadi, M. Novzari, E. Nikname, M.M. Golshan, Coommun.Theor. Phys. 2014, 61, 765 .

[5] Y. Yakar, B. Çakır, A. Özmen, Int. J. Mod. Phys. C 2007, 18, 61.

[6] H. Kes, A. Bilekkaya, S. Aktas, S. Okan, Superlatt. and Microstruct. 2017, 111, 966.

[7] O. Akankan, I. Erdogan, H. Akbaş, Physica E 2006, 35 217;

A.R. Jafari, Y. Naimi, S. Davatolhagh, Opt. Quant. Electron 2013, 45, 517.

[8] X.C. Li, C.B. Ye, J. Gao, B. Wang, Chin. Phys. B 2020, 29, 087302;

J. D. Castano-Yepes, A. Amor-Quiroz, C.F. Ramirez-Gutierrez, E.A. Gomez, Physica E 2020, 109, 59.

[9] H. El Ghazi,A. Joroi, I. Zorkani, E. Feddi, A. El Mouchtachi, Physica B 2018, 537, 207;

E. Niculescu, C. Stan, M. Cristea, C. Trusca, Chem. Phys. 2017, 493, 32.

[10] B. Çakir, Y. Yakar, A. Özmen, Chem. Phys. Lett. 2017, 684, 250;

Y. Yakar, B. Çakir, A. Özmen, Chem. Phys. 2018, 513, 213.

[11] A.J. Peter, N. Karthikeyan, C.W. Lee, J. Electronic Materials 2020, 49, 2257;

S. Nasa, S.P. Purohit, Physica E, 2020, 118, 113913.

[12] K.A. Rodriguez- Megdaleno, R. Perez-Alvarez, J.C. Martinez-Orozco, J. Alloys and Compounds, 2018, $736,211$.

[13] E.B. Al, E. Kasapoğlu, S. Sakiroğlu, H. Sarı, İ. Sökmen, C.A. Duque, Physica E, 2020, 119, 114011; S. Antil, M. Kumar, S. Lahon, S. Dahiya, A. Ohlan, R. Puna, A.S. Maan, Physica B 2019, 552, 202.

[14] D. Makhlouf, M. Choubani, F. Saidi, H. Maaref, Physica E 2018, 103, 87. 
[15] S. Pal, M. Ghosh, C.A. Duque, Philosophical Magazine 2019, 99, 2457.

[16] M. Amini, M. Soleimani, M.H. Ehsani, Superlatt. Microstruct. 2017, 112, 680.

[17] Y. Yakar, B. Cakir, A. Ozmen, Superlatt. Miceostruct. 2013, 60, 389; Y. Yakar, B. Cakir, A. Ozmen, Chem Phys. Lett. 2017, 684, 250.

[18] D.N. Thao, L.T.N. Bao, D.D. Phuoc, N.H. Quang, Semicond. Sci. Technol. 2017, 32, 025014.

[19] A. Gil-Corrales, A.L. Morales, R.L. Restrepo, M.E. Mora-Ramos, C.A. Duque, Physica B 507 (2017) 79; L. Stevanović, N. Filipović, V. Pavlović, Optical Materials 2019, 91, 62.

[20] Y. Yakar, B. Çakır, A. Özmen, Chem. Phys. Lett. 2018, 708, 138; Y. Yakar, B. Çakır, U. Atav, A. Özmen, Chem . Phys. 2016, 475, 61.

[21] J.S. Huang, G.X. Chen, Int. J. Modern Phys. B, 2018, 32, 1850202, F.S. Nammas, Physica Scripta 2020, 95, 015802 .

[22] E. Kasapoglu, C.A. Duque, M.E. Mora-Ramos, R.L. Restrepo, F. Ungan, U. Yesilgul, H. Sarı, I. Sokmen, Mater. Chem. Phys. 2015, 154, 170.

[23] A. Naifar, N. Zeiri, S.A.B. Nasrallah, M. Said, Photonics and Nanostructures - Fundamentals and Applications 2020, 40 , 100789.

[24] R. Khordad, N. Fathizadeh, S. Davatolhagh, A.R. Jafari, Eur. Phys. J.B 2012, 85, 353; X.Q. Wang, Y.J. Chen, J.L. Xiao, Int. J. Modern Phys. 2019, 33, 1950239.

[25] Y. Yakar, B. Çakır, A. Özmen, Comput. Phys. Commun. 2015, 188, 88; B. Cakir, Y. Yakar, A. Özmen Physica B 2017, 510, 86.

[26] S.M. Arif, A. Bera, A. Ghosh, M. Ghosh, Physica B 2020, 588, 412166; A. Ghosh, A. Bera, S. Saha, S.M. Arif, M. Ghosh, Superlatt. Microstruct. 2018, 114, 259.

[27] Y. Karaaslan, B. Gisi, S. Şakiroğlu, E. Kasapoğlu, H. Sarı, İ. Sökmen, Phys. Lett A 2018, 382, 507; M. Solaimani, D.H.T. Tehrani, J. Comput. Electronic 2010, 19, 160.

[28] S. Saha, S. Pal, J. Ganguly, M. Ghosh, J. Adv. Phys. 2017, 6 48; S.M. Arif, A. Ghosh, A. Bera, M. Ghosh, J. Phys. Chem. Solids 2018, 121, 51.

[29] J.M. Jacob, R. Rajan, M. Aji, G.G. Kurup, A. Pugazhendhi, Ceramics International 2019, 45, 48574862; S.L. Talwar, S. Lumb, K.D. Sen, V. Prasad, Physica Scripta 2020, 95, 035404.

[30] Y. Yakar,B. Çakır, A. Özmen, J. Lumin. 2013, 134, 778; A. Özmen, B. Çakır, Y. Yakar, J. Lumin. 2013, 137, 259.

[31] A. Aquino, J. Garza, A. Flores-Riveros, J.F. Rivas-Silva, K.D. Sen, The J. Chem. Phys. 2006, 124, 054311.

[32] C.L. Wilson, H.E. Montgomery, K.D. Sen, D.C. Thompson, Phys. Lett. A 2010, 374, 4415.

[33] E.V. Ludeña, J. Chem. Phys. 1978, 69, 1770.

[34] J. Garza, J. M. H. Pérez, J. Z. Ramírez, R. Vargas, J. Phys. B: At. Mol. Opt. phys. 2012, 45, 015007.

[35] Y. Yakar, B. Çakır, A. Özmen, Phil. Magazine 2015, 95, 311; Y. Yakar, B. Çakır, A. Özmen, Int. J. Quant. Chem, 2011, 111, 4139.

[36] A. Flores-Riveros, N. Aquino, H.E. Montgomery, Phys. Lett. A 2010, 374, 1246.

[37] A. Sarsa, C.L. Sech, J. Chem. Theor. Comput. 2011, 7, 2786. 
[38] A.D. Sañu-Ginarte, E.M. Gullién-Romero, L. Ferrer-Galindo, L.A. Ferrer-Moreno, R. Betacourt-Riera, R. Riera, Results in Physics 2019, 13, 102261; A.D. Sañu-Ginarte, L. Ferrer-Galindo, R.A. Rosas, A. CorellaMadueno, R. Betacourt-Riera, L.A. Ferrer-Moreno, R. Riera, J. Phys. Commun. 2018, 2, 015001.

[39] C. Martinez-Flores, R. Cabrera-Trujilo, Matter and Radiat. Extremes 2020, 5, 024401.

[40] Y. Yakar, Chin. J. Chem. 2007, 25, 25; Y. Yakar, A. Özmen, Ü. Atav, Chin. J. Chem. 2006, 24, 603.

[41] L. Stevanović, J. Phys. B: At. Mol. Opt. Phys. 2010, 43, 165002.

[42] S. Lumb, S. Lumb, V. Prasad, Eur. Phys. J. Plus 2015, 130, 149.

[43] H.E. Montgomery Jr, K.D. Sen, Phys. Lett. A 2012, 376, 1992.

[44] O. Motopan, S. A. Ndengue, K. D. Sen, Int. J. Quant. Chem 2011, 111, 4425.

[45] J.G. Kirkwood, Phys. Z. 1932, 33, 57.

[46] A.Z. Tang, F. T. Chan, Phys. Rev. A 1986, 33, 3671.

[47] J.L. Marin, S. A. Cruz, J. Phys. B: At. Mol. Opt. Phys. 1992, 25, 4365.

[48] R.N. Barnett, P.J. Reynolds, W.A. Lester, Jr., Int. J. Quantum Chem 1992, 42, 837.

[49] T. Koga, H. Tatewaki, A. J. Thakkar, Theor. Chim. Acta. 1993, 86, 477.

[50] R.N. Barnet, E.M. Johnson, W.A. Lester, Phys. Rev. A 1995, 51, 2049.

[51] A.W. Weiss, Cand. J . Chem. 1992, 70, 456. 


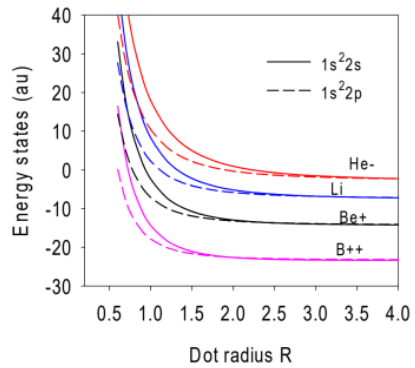

Fig.1 Ground and excited state energy of lithium-like dots versus dot radius. 

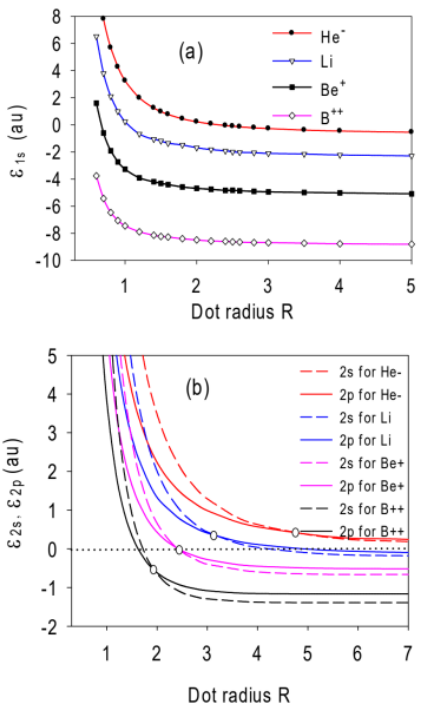

Fig.2 Orbital energies of lithium-like dots versus dot radius: (a) for $\epsilon_{1 s}$, (b) for $\epsilon_{2 s}$ and $\epsilon_{2 p}$ orbital. 


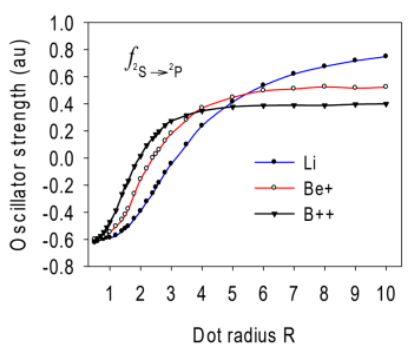

Fig.3 Oscillator strength for the dipole transition between $1 s^{2} 2 s\left({ }^{2} S\right)$ and $1 s^{2} 2 p\left({ }^{2} \mathrm{P}\right)$ levels of lithium-like dots versus dot radius. 

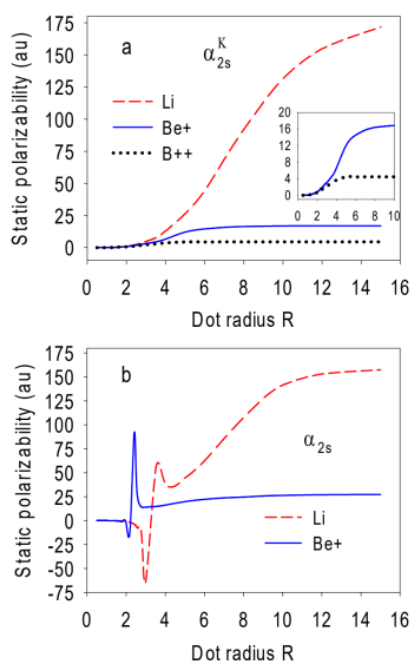

Fig.4 Static polarizability of lithium-like dots versus dot radius: (a) from the Kirkwood expression, (b) from the oscillator strength. 


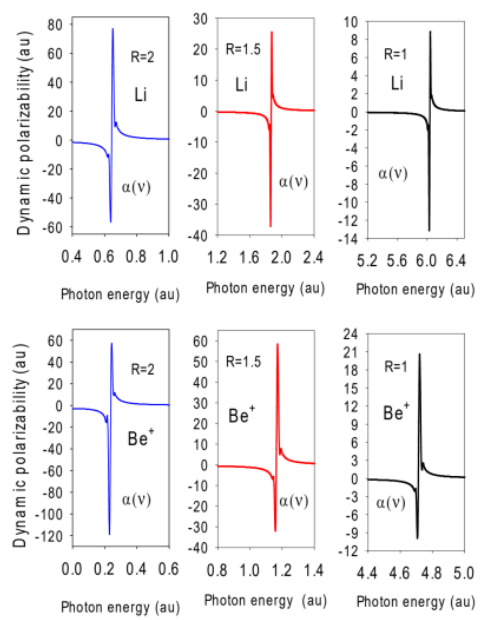

Fig.5 Dynamic polarizability of $L i$ and $B e^{+}$dots versus photon energy $h v$ for three different dot radius: $\mathrm{R}=1,1.5$ and 2 . 

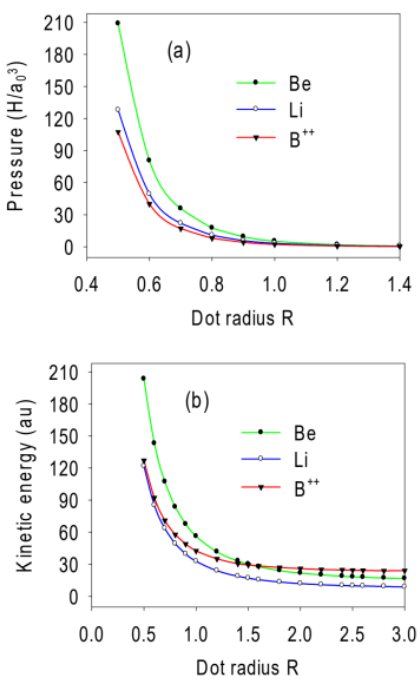

Fig.6 Static pressure induced by cavity in (a) and total kinetic energy in (b) versus dot radius for $\mathrm{Li}, \mathrm{B}^{++}$and $\mathrm{Be}$ dots.

\section{Hosted file}

Table 1.docx available at https://authorea.com/users/358081/articles/480384-energystates-oscillator-strengths-and-polarizabilities-of-many-electron-atoms-confined-byan-impenetrable-spherical-cavity

\section{Hosted file}

Table 2.docx available at https://authorea.com/users/358081/articles/480384-energystates-oscillator-strengths-and-polarizabilities-of-many-electron-atoms-confined-byan-impenetrable-spherical-cavity

\section{Hosted file}


Table 3.docx available at https://authorea.com/users/358081/articles/480384-energystates-oscillator-strengths-and-polarizabilities-of-many-electron-atoms-confined-byan-impenetrable-spherical-cavity

\section{Hosted file}

Table 4.docx available at https://authorea.com/users/358081/articles/480384-energystates-oscillator-strengths-and-polarizabilities-of-many-electron-atoms-confined-byan-impenetrable-spherical-cavity

\section{Hosted file}

Table 5.docx available at https://authorea.com/users/358081/articles/480384-energystates-oscillator-strengths-and-polarizabilities-of-many-electron-atoms-confined-byan-impenetrable-spherical-cavity

\section{Hosted file}

Table 6.docx available at https://authorea.com/users/358081/articles/480384-energystates-oscillator-strengths-and-polarizabilities-of-many-electron-atoms-confined-byan-impenetrable-spherical-cavity 